\title{
Nanoparticles in Gastrooncology
}

\author{
André Jefremow Markus F. Neurath \\ Department of Internal Medicine 1, Friedrich-Alexander-Universität Erlangen-Nürnberg (FAU), Erlangen, Germany
}

\section{Keywords}

Nanomedicine · Nanoparticles · Gastrooncology · Gastrointestinal tumors

\section{Abstract}

Background: Gastrointestinal malignancies have the greatest incidence and cancer-associated death rates worldwide. Routine therapeutic modalities include surgery, chemotherapy and radiation but they often fail to reach the goal of cancer-free survival. Summary: In the light of this urgent medical need for the treatment of Gl tumors, nanotechnology-based approaches, i.e. nanomedicine, promise new therapeutic options. Using nanoparticles instead of classically designed drugs, targeting anticancer agents directly to the tumor site may revolutionize both diagnostic and therapeutic tools thereby facilitating the identification and elimination of malignant cells. Importantly, diagnostic insight and therapeutic effects can be achieved simultaneously through the same nanoparticle. Additionally, a nanoparticle may be loaded with more than one agent, thereby further increasing the value and power of the nanotechnology approach in oncologic therapeutic concepts. Although most insight into mechanisms of nanomedicine has been gained from in vitro and preclinical in vivo models, few clinical trials have been conducted, and nanomedicine-based concepts are already part of standard treatment algorithms. However, despite substantial progress it remains a challenge to design nanoparticles that feature all desirable characteristics at the same time. Key Messages: This review seeks to provide substantial insight into the current status of nanomedicinebased approaches employed for diagnostic and/or therapeutic purposes in the field of gastrointestinal cancers by highlighting achievements and pointing out unresolved issues that need to be further addressed by future research attempts.

\footnotetext{
(c) 2020 S. Karger AG, Basel
}

\section{Introduction}

Globally, gastrointestinal (GI) tumors are the leading cause for cancer-related death and show the highest incidence. Among those, colorectal cancer, gastric cancer, hepatocellular carcinoma (HCC) and pancreatic cancer are the most frequently observed solid tumors [1]. Even though many attempts to reduce their disease burden have been undertaken, GI tumors are still overall associated with a poor outcome due to both delayed diagnosis in an already incurable state and insufficient therapeutic options in the light of a heterogeneous and so far only partially resolved tumor biology. Accordingly, established treatment regimens consisting of surgery, systemic chemo- and/or immunotherapy and/or local radiotherapeutic measures routinely fail to ultimately cure the majority of patients in advanced tumor stages.

Under the term nanomedicine, nanotechnologybased approaches including designing tumor-targeting nanoparticles are summarized. Nanoparticles represent a very heterogeneous group of particles ranging from 1 to $100 \mathrm{~nm}$ in diameter. Their components can be organic or inorganic, and they show different shapes [2]. Currently, mainly gold nanoparticles, magnetic nanoparticles, quantum dots, fluorescence-labeled nanoparticles, graphenes and graphene oxides, and dendrimers and stimulus-responsive polymers are used in research [3]. Nanoparticles can reach their target by an enhanced permeability and retention effect. This effect results from unstructured tumor vessels leaving pores into which nanoparticles can accumulate. Another approach to guide nanoparticles to their target is binding their surface to tumor-specific ligands like antibodies. Nanoparticles as a drug-delivering system are usually characterized by a long circulation time and are protected by liver and kidney metabolism [3]. However, also alternative 
Table 1. Important current and finished clinical trials using nanoparticles

\begin{tabular}{lllll}
\hline Drug & Nanoparticle type & Status & Disease & Reference \\
\hline MTL-CEBPA & Lipid & Phase 1 & HCC & NCT02716012 \\
\hline DCR-MYC & Lipid & Phase 1/2 & HCC & NCT02314052 \\
\hline Irinotecan & Liposome & Phase 1/2 & $\begin{array}{l}\text { Gastric cancer or gastroesophageal junction } \\
\text { adenocarcinoma }\end{array}$ & NCT03739801 \\
\hline Irinotecan & Liposome & Phase 1/2 & $\begin{array}{l}\text { Metastatic biliary tract carcinoma, metastatic colorectal } \\
\text { carcinoma, metastatic gastroesophageal junction }\end{array}$ & NCT03337087 \\
& & & adenocarcinoma, metastatic pancreatic adenocarcinoma & NCT01437007 \\
\hline TKM 080301 & Lipid nanoparticle & Phase 1 & $\begin{array}{l}\text { Secondary liver cancer } \\
\text { HCC }\end{array}$ & NCT02191878 \\
\hline Paclitaxel & Albumin & FDA/EMA & Pancreatic cancer & 55, 56 \\
\hline AZD4635 & Nanoparticle suspension & Phase 1 & Advanced solid malignancies & NCT02740985 \\
\hline Doxorubicin & $\begin{array}{l}\text { Doxorubicin docetaxel } \\
\text { pluronic block copolymers }\end{array}$ & Phase 2 & Gastroesophageal adenocarcinoma & 62 \\
\hline
\end{tabular}

HCC, hepatocellular carcinoma; FDA, Food and Drug Administration; EMA, European Medicines Agency.

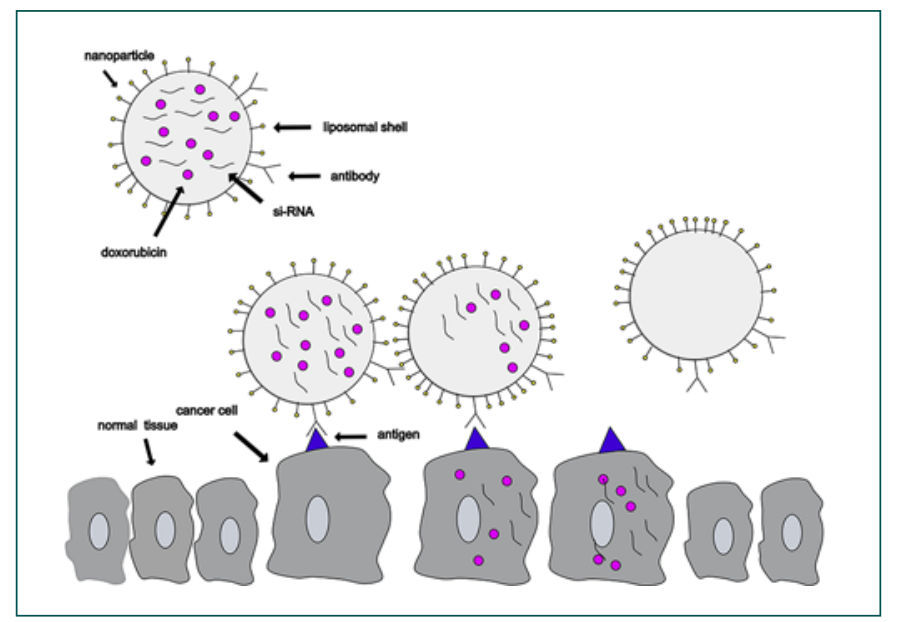

Fig. 1. Example of a nanoparticle working as a nanocarrier: a liposomal nanoparticle is loaded with doxorubicin and siRNA in its core. It is connected to an antibody. The nanoparticle detects the cancer cell via antibody-antigen contact and delivers doxorubicin and siRNA.

application modes have been employed such as direct delivery to the intestinal tumors through an endoscopically guided instillation [4].

Different nanoparticles show distinct characteristics. For example, superparamagnetic nanoparticles can serve as contrast agents in magnetic resonance imaging (MRI). They can also be used as treatment tools when drugs or agents like nucleic acid are bound to them or encapsulated by them (Fig. 1). But nanoparticles can be treatment themselves [2,5-8]. For example, gold nanoparticles and other nanoparticles are utilized for hyperthermia and photodynamic therapy after irradiation with light in the infrared region [9]. There is a growing body of literature that recognizes the importance of nanomedicine, yet research takes place mainly in vitro and in vivo, but some agents have already been tested in clinical trials (Table 1 ) or are yet part of standard cure $[2,5-8]$.

\section{Nanoparticles in Colorectal Cancer}

Colorectal cancer (CRC) belongs to the tumors with the highest incidence and mortality in the world. Besides the standard approaches in therapy like surgery, chemotherapy and radiation, great efforts have been made in the field of nanomedicine lately. Evidence from a number of experimental studies has established that nanoparticles represent a feature in treating CRC.

LE-SN38 is a liposomal formulation of SN38, the active metabolite of CPT-11 (irinotecan). This nanoparticle showed greater tumor growth inhibition than CPT-11 in a xenograft model with SCID (severe combined immunodeficiency) mice [10]. Thermodox is used to treat liver metastases, which derive from CRC. It is a liposome that delivers doxorubicin after thermal stimulation, and its use is combined with radiofrequency thermal ablation [8]. Both, LE-SN38 and Thermodox are tested in clinical studies.

CPX-1, a liposome-encapsulated formulation of irinotecan and floxuridine has already completed phase II clin- 
ical trials in patients, who had been treated with oxaliplatin or irinotecan previously. Out of 15 patients, 9 showed stable disease, 2 had partial response and only 2 developed progressive disease. Two patients could unfortunately not be evaluated. Six patients showed a progression-free survival of more than 6 months [11].

Yang et al. [12] developed oxaliplatin long-circulating liposomes (pegylated-liposomal oxaliplatin). They could demonstrate that their use was associated with a higher amount of apoptosis in tumors in comparison with free oxaliplatin in vivo using a xenograft mouse model for CRC.

First steps to directly target CRC metastases were performed in an experiment with core/pegylated shell nanoparticles, which delivered DNA for gene therapy. The nanoparticles selectively transfected CRC metastases in vivo, even though only a small fraction of the cells expressed the transgene [13].

The challenge of targeting only the tumor site has been addressed in different experiments.

Of particular concern is A33. The A33 antigen plays a pivotal role in targeting CRC. Its expression in about 95\% of all colorectal tumors makes the humanized A33 monoclonal antibody an attractive guide to deliver nanoparticles to the tumor site [14]. The binding to polymer capsules formed by the layer-by-layer method gives the opportunity to deliver drugs and other anticancer agents directly to the tumor [15]. But not only antibodies also peptides can function as a targeting aim.

One study from Gounaris et al. [16] set out to investigate the usefulness of G3-C12 for detecting galectin-3 and G11 to target epidermal growth factor receptor on fluorescently labeled $\mathrm{N}$-(2-hydroxypropyl)methacrylamide copolymers. Furthermore, they connected G11-binding nanoparticles to doxorubicin creating a tumor-specific drug-delivering system.

Besides therapeutical use of nanoparticles, these particles have emerged as powerful platforms for diagnostic approaches. Gounaris et al. [16] were able to identify dysplastic foci within chronically inflamed colons using endoscopic fluorescence imaging in IL-10 $10^{-/-}$colitic mice. Through the utilization of lectin-conjugated Fe2O3@Au core@shell nanoparticles, He et al. [17] performed dualmodality imaging to detect colorectal cancer in nude mice in T2-weighted MRI and computer tomography.

\section{Nanoparticles in Gastric Cancer}

Determining the impacts of nanomedicine on gastric cancer is important for the future of diagnostics and cancer treatment. Besides that, many research groups do pioneer work combining them, which is named theranostic [18].
Recent research has reported its use in different diagnostic tools such as MRI, fluorescence imaging and endoscopy [7].

Wang et al. [19] used superparamagnetic iron oxide nanoparticles (SPION) for in vitro and in vivo experiments. They coated it with $\mathrm{dSiO}_{2}$ and after labeling with near infrared fluorescence dye and anti-CD146 monoclonal antibody it led to a nanoparticle called 800ZW-SPION@dSiO2-YY146. This nanoparticle was used for in vitro and in vivo imaging. They performed $\mathrm{MR} /$ near infrared fluorescence imaging using 800ZW-SPION@ dSiO2-YY146 in an MKN45 xenograft tumor model showing its ability to detect gastric cancer by targeting the tumor marker CD146 [19].

Few studies concentrate on nonsupramagnetic nanoparticles. Folic acid-conjugated silica-capped gold nanoclusters designed by Zhou et al. [20] were able to detect FR(+) MGC803 cells in a nude mouse model showing red emitting fluorescence optical property with X-ray absorbance for optical and computed tomography dualmodality imaging of gastric cancer.

Previous research has established the design and implementation of glucose-regulated protein 78 (GRP78)guided polymeric micelles to diagnose gastric cancer. GRP78 is considered to be a reliable gastric cancer biomarker showing an increased expression level on the cell surface of gastric cancer cells. Cheng et al. [21] coupled the micelles to indium-111 ( $\left.{ }^{111} \mathrm{In}\right)$. Tumor imaging with nano single photon emission computed tomography/ computed tomography revealed higher radioactive intensity of GRP78-binding protein ${ }^{111}$ In-labeled micelles in comparison to ${ }^{111}$ In-labeled micelles without coupled GRP78-binding protein.

Recent studies by Wang et al. [22] included tumor detection in a xenograft tumor model for esophagus cancer in rats using surface-enhanced Raman scattering nanoparticles conjugated to human epidermal growth factor receptor 2 or epidermal growth factor receptor. These nanoparticles were administered orally and endoscopy with multimode fibers for illuminating and detection was performed. Hereby, tumors could not only be detected but the amount of human epidermal growth factor receptor 2 and epidermal growth factor receptor could be quantified.

Theranostic describes the combination of diagnostic and therapy. There are two different manners of it. On the one hand imaging can detect the therapeutic nanoparticle, and on the other hand diagnostic and therapeutic nanoparticles can be administered together [23-26]. Huang et al. [27, 28] designed photosensitizerconjugated carbon dots called C-dots-Ce6. They combine tumor homing with near infrared fluorescence imaging-guided photodynamic treatment. Additionally, drug agents could be magnetically led to the tumor, and 
the magnetic component of the nanoparticle made it visible in MRI.

An innovative technique to deliver drugs using pegylated polycaprolactone nanoparticles containing gelatinase-sensitive peptide was developed by the group of Baorui Liu. Since gelatinases are overexpressed in gastric cancer [29-31], the nanoparticles can target the cancer cells easily and deliver different kinds of agents like chemotherapeutics, small molecules or nucleic acids [3242].

Besides that, other efforts were undertaken using nanoparticles for treating gastric cancer. SP1049C (Pluronic L61, F127-doxorubicin), a P-glycoprotein targeting micellar formulation of doxorubicin, showed good results in a clinical phase II trial. The objective response rate was $47 \%$ (95\% CI: $24.4-71$ ) in the evaluable patient population [43].

\section{Nanoparticles in HCC}

First steps for the treatment of HCC with nanomedicine were made almost three decades ago. In 1991 Duncan and colleagues [44] developed two different N-(2hydroxypropyl)methacrylamide copolymers containing doxorubicin and galactosamine, which target hepatocyte galactose receptor, named PK1 and PK2. PK2 targets asialoglycoprotein receptor that is overexpressed in HCC. Patients reported the same side effects with PK2 and unformulated doxorubicin. Asialoglycoprotein receptor expression levels become lower during disease progression suggesting asialoglycoprotein receptor expression as a biomarker for treatment with this agent [6,44-46].

$\mathrm{Xu}$ et al. [47] developed a new nanoparticle carrying doxorubicin, and it shows a low toxicity profile in vivo while being very selective.

A different approach was used by Devulapally et al. [48], when they co-encapsulated gemcitabine and antisense-microRNA-21 in pegylated-poly(lactic) coglycolic acid nanoparticles achieving treatment with two different agents, which was more successful than each agent alone in vitro.

Several studies have investigated the application of small interfering RNAs (siRNA) for HCC treatment [6]. In the context of nanomedicine, Wang et al. [49] designed a nanovector (NP-siRNA-GPC3 antibody) with an iron core and coated with chitosan-polyethylene glycol-grafted polyethylene imine copolymer. This nanovector is conjugated with a monoclonal antibody against human glypican-3 receptor, which shows high expression levels in HCC and transports siRNA. Sun et al. [50] constructed a polyethylene imine-modified liposome, which transports siRNA targeting glypican-3 and sorafenib, a mulltikinase inhibitor, which is a standard drug for HCC treatment with partially serious side effects. There are several other studies going on in vitro and in vivo testing the different characteristic potentials of nanoparticles including the delivery of nucleic acids, drug agents or providing endogenous toxicity. Recent research reported about a thermosensitive liposomal formulation named Thermodox, which forms holes after being heated up to $40-45^{\circ} \mathrm{C}$ leading to the release of a loaded drug with promising results in a phase III clinical trial [51]. CEBPA (CCAA/enhancer-binding protein $\alpha$ ), a regulator in hepatic function, is downregulated in HCC. Coating activating RNA in a liposomal nanoparticle and administering it in a rodent HCC model reduced tumor burden, so this formula called MTL-CEBPA was further tested in clinical trials $[52,53]$. The overexpression of Polo-like kinase 1 in HCC led to a phase I clinical trial using siRNA encapsulated in lipid nanoparticles. Due to limited effects no further studies in HCC are planned [54]. However, this substance is now being investigated for the treatment of liver metastases (NCT01437007).

\section{Nanoparticles in Pancreatic Cancer}

Pancreatic cancer belongs to the cancer types in which nanoparticles are already part of standard treatment. It is now well established from a variety of studies that the albumin-bound nanoparticle nab-paclitaxel (abraxane) is part of first-line treatment in unresectable and metastatic disease [55, 56].

Gemcitabine is another standard chemotherapeutic in pancreatic cancer. Patra et al. [57] designed a gold nanoparticle that delivers gemcitabine and used cetuximab (a monoclonal epidermal growth factor antibody) to guide it to the tumor site. In this experiment, tumor inhibition was increased in vitro and in vivo.

A key issue in treating pancreatic cancer is the development of resistance to gemcitabine due to CD47, which provides an antiphagocytosis signal. Therefore, gemcitabine has been coupled to iron oxide magnetic nanoparticles that include an anti-CD47 antibody. This formula inhibited tumor growth in vivo [58]. Hoskins et al. [59] used coupled bisnaphthalimidopropyldiaaminooctane, a novel drug against pancreatic cancer with poor water solubility, to polyallylamine grafted with $5 \%$ mole cholesteryl pendant groups. So, the drug is discharged through holes in the shell. In vitro this nanoparticle showed a higher cytotoxic effect on pancreatic cancer cells than gemcitabine.

There are different approaches using metal and metal oxide nanoparticles with antipancreatic cancer activity [5]. For example, cerium oxide nanoparticles sensitize cancer cells to radiation, when administered before treatment while being nontoxic to normal tissue [60]. Pal et al. 
[61] designed plectin-1-pancreatic targeting peptidegold nanoparticles, which deliver gemcitabine to pancreatic cancer. It has a huge advantage due to its selectivity in vivo.

\section{Conclusion}

Nanomedicine has the potential to improve diagnostic tools and increase therapeutic options for GI cancers. The heterogeneity of available nanoparticles seems to provide a great advantage, for there are many different options going along with it like binding and encapsulating drugs, different agents and target-directed therapy. Some nanoparticles are already in clinical use. The delivery of one or more anticancer drugs directly to the tumor site and the fact that some nanoparticles can be used for imaging make them very attractive. Published results from in vitro and in vivo research are indeed promising. Yet, some limitations must be taken into account. First, most research on nanoparticles has been performed in vitro and in vivo, while clinical data are rarely available. Hence, the efficacy of individual nanoparticles must be evaluated in clinical studies. In fact, some nanoparticles with promising results in vitro and in vivo failed in clinical tests. Similarly, data on safety and toxicity of distinct nanoparticles are sparse. Another negative aspect considers the costs as well as the complexity of the designing and manufacturing under controlled pharmaceutical production conditions. Still, nanomedicine is an interesting and promising field in medical science. Since it deals with medicine, physics, pharmacology and chemistry there is a huge need for multidisciplinary research.

\section{Disclosure Statement}

The authors have no conflicts of interest to declare. No funding was received.

\section{Author Contributions}

A.J.: writing - original draft; writing - review and editing. M.F.N.: writing - original draft; writing - review and editing.

\section{References}

1 Bray F, Ferlay J, Soerjomataram I, Siegel RL, Torre LA, Jemal A. Global cancer statistics 2018: GLOBOCAN estimates of incidence and mortality worldwide for 36 cancers in 185 countries. CA Cancer J Clin. 2018 Nov;68(6): 394-424.

2 Singh M, Harris-Birtill DC, Markar SR, Hanna GB, Elson DS. Application of gold nanoparticles for gastrointestinal cancer theranostics: A systematic review. Nanomedicine (Lond). 2015 Nov;11(8):2083-98.

3 Manzur A, Oluwasanmi A, Moss D, Curtis A, Hoskins C. Nanotechnologies in pancreatic cancer therapy. Pharmaceutics. 2017 Sep; 9(4):223-39.

4 Zavaleta CL, Garai E, Liu JTC, Sensarn S, Mandella MJ, Van de Sompel D, et al. A Raman-based endoscopic strategy for multiplexed molecular imaging. Proc Natl Acad Sci USA. 2013 Jun;110(25):E2288-97.

5 El-Zahaby SA, Elnaggar YS, Abdallah OY. Reviewing two decades of nanomedicine implementations in targeted treatment and diagnosis of pancreatic cancer: an emphasis on state of art. J Control Release. 2019 Jan;293:21-35.

6 Elnaggar MH, Abushouk AI, Hassan AHE, Lamloum HM, Benmelouka A, Moatamed $\mathrm{SA}$, et al. Nanomedicine as a putative approach for active targeting of hepatocellular carcinoma. Semin Cancer Biol. 2019 Aug;293: 21-35.

7 Li R, Liu B, Gao J. The application of nanoparticles in diagnosis and theranostics of gastric cancer. Cancer Lett. 2017 Feb;386(1):123-30.

8 Cisterna BA, Kamaly N, Choi WI, Tavakkoli A, Farokhzad OC, Vilos C. Targeted nanoparticles for colorectal cancer. Nanomedicine (Lond). 2016 Sep;11(18):2443-56.
9 Akhter S, Ahmad MZ, Ahmad FJ, Storm G, Kok RJ. Gold nanoparticles in theranostic oncology: current state-of-the-art. Expert Opin Drug Deliv. 2012 Oct;9(10):1225-43.

10 Lei S, Chien PY, Sheikh S, Zhang A, Ali S, Ahmad I. Enhanced therapeutic efficacy of a novel liposome-based formulation of SN-38 against human tumor models in SCID mice. Anticancer Drugs. 2004 Sep;15(8):773-8.

11 Batist G, Gelmon KA, Chi KN, Miller WH, Chia SKL, Mayer LD, et al. Safety, pharmacokinetics, and efficacy of CPX-1 liposome injection in patients with advanced solid tumors. Clin Cancer Res. 2009 Jan;15(2):692700.

12 Yang C, Liu HZ, Fu ZX, Lu WD. Oxaliplatin long-circulating liposomes improved therapeutic index of colorectal carcinoma. BMC Biotechnol. 2011 Mar;11(1):21.

13 Yang J, Hendricks W, Liu G, McCaffery JM, Kinzler KW, Huso DL, et al. A nanoparticle formulation that selectively transfects metastatic tumors in mice. Proc Natl Acad Sci USA. 2013 Sep;110(36):14717-22.

14 Chong G, Lee FT, Hopkins W, Tebbutt N, Cebon JS, Mountain AJ, et al. Phase I trial of 131I-huA33 in patients with advanced colorectal carcinoma. Clin Cancer Res. 2005 Jul;11(13):4818-26.

15 Cortez C, Tomaskovic-Crook E, Johnston AP, Scott AM, Nice EC, Heath JK, et al. Influence of size, surface, cell line, and kinetic properties on the specific binding of A33 antigen-targeted multilayered particles and capsules to colorectal cancer cells. ACS Nano. 2007 Sep;1(2):93-102.
16 Gounaris E, Martin J, Ishihara Y, Khan MW, Lee G, Sinh P, et al. Fluorescence endoscopy of cathepsin activity discriminates dysplasia from colitis. Inflamm Bowel Dis. 2013 Jun; 19(7):1339-45.

17 He X, Liu F, Liu L, Duan T, Zhang H, Wang Z. Lectin-conjugated Fe2O3@Au core@Shell nanoparticles as dual mode contrast agents for in vivo detection of tumor. Mol Pharm. 2014 Mar;11(3):738-45.

18 Huang $\mathrm{P}$, Lin J, Wang X, Wang Z, Zhang C, $\mathrm{He} M$, et al. Light-triggered theranostics based on photosensitizer-conjugated carbon dots for simultaneous enhanced-fluorescence imaging and photodynamic therapy. Adv Mater. 2012 Sep;24(37):5104-10.

19 Wang P, Qu Y, Li C, Yin L, Shen C, Chen W, et al. Bio-functionalized dense-silica nanoparticles for MR/NIRF imaging of CD146 in gastric cancer. Int J Nanomed. 2015 Jan;10:74963.

20 Zhou Z, Zhang C, Qian Q, Ma J, Huang P, Zhang $\mathrm{X}$, et al. Folic acid-conjugated silica capped gold nanoclusters for targeted fluorescence/X-ray computed tomography imaging. J Nanobiotechnol. 2013 May;11(1): 17.

21 Cheng CC, Huang CF, Ho AS, Peng CL, Chang CC, Mai FD, et al. Novel targeted nuclear imaging agent for gastric cancer diagnosis: glucose-regulated protein 78 binding peptide-guided 111In-labeled polymeric micelles. Int J Nanomed. 2013;8:1385-91.

22 Wang YW, Kang S, Khan A, Bao PQ, Liu JTC. In vivo multiplexed molecular imaging of esophageal cancer via spectral endoscopy of topically applied SERS nanoparticles. Biomed Opt Express. 2015 Oct;6(10):3714-23. 
23 Shubayev VI, Pisanic TR, Jin S. Magnetic nanoparticles for theragnostics. Adv Drug Deliv Rev. 2009 Jun;61(6):467-77.

24 Wittrup A, Zhang S-H, Svensson KJ, Kucharzewska P, Johansson MC, Mörgelin M, et al. Magnetic nanoparticle-based isolation of endocytic vesicles reveals a role of the heat shock protein GRP75 in macromolecular delivery. Proc Natl Acad Sci USA. 2010 Jul;107(30): 13342-7.

25 Lima-Tenório MK, Pineda EA, Ahmad NM, Fessi H, Elaissari A. Magnetic nanoparticles: in vivo cancer diagnosis and therapy. Int J Pharm. 2015 Sep;493(1-2):313-27.

26 Gobbo OL, Sjaastad K, Radomski MW Volkov Y, Prina-Mello A. Magnetic Nanoparticles in Cancer Theranostics. Theranostics. 2015 Sep;5(11):1249-63.

27 Huang P, Li Z, Lin J, Yang D, Gao G, Xu C, et al. Photosensitizer-conjugated magnetic nanoparticles for in vivo simultaneous magnetofluorescent imaging and targeting therapy. Biomaterials. 2011 May;32(13):3447-58.

28 Huang $\mathrm{P}$, Lin J, Wang X, Wang Z, Zhang C, $\mathrm{He} \mathrm{M}$, et al. Light-triggered theranostics based on photosensitizer-conjugated carbon dots for simultaneous enhanced-fluorescence imaging and photodynamic therapy. Adv Mater. 2012 Sep;24(37):5104-10.

29 Dragutinović VV, Radovanović NS, IzraelZivković LT, Vrvić MM. Detection of gelatinase B activity in serum of gastric cancer patients. World J Gastroenterol. 2006 Jan;12(1): 105-9.

30 Mroczko B, Lukaszewicz-Zając M, Gryko M, Kędra B, Szmitkowski M. Clinical significance of serum levels of matrix metalloproteinase 2 (MMP-2) and its tissue inhibitor (TIMP-2) in gastric cancer. Folia Histochem Cytobiol. 2011;49(1):125-31.

31 Nomura Y, Takasaki K, Tada M, Yoshimoto M, Ishida H, Murata N, et al. Pro-MMP-9 is associated with poor prognosis in gastric cancer. Scand J Gastroenterol. 2007 Sep;42(9): 1070-2.

32 Li R, Wu W, Liu Q, Wu P, Xie L, Zhu Z, et al. Intelligently targeted drug delivery and enhanced antitumor effect by gelatinase-responsive nanoparticles. PLoS One. 2013 Jul; 8(7):e69643.

33 Li R, Li X, Xie L, Ding D, Hu Y, Qian X, et al. Preparation and evaluation of PEG-PCL nanoparticles for local tetradrine delivery. Int J Pharm. 2009 Sep;379(1):158-66.

34 Wang Q, Wu P, Ren W, Xin K, Yang Y, Xie C, et al. Comparative studies of salinomycinloaded nanoparticles prepared by nanoprecipitation and single emulsion method. $\mathrm{Na}$ noscale Res Lett. 2014 Jul;9(1):351.

35 Liu Q, Li RT, Qian HQ, Wei J, Xie L, Shen J, et al. Targeted delivery of miR-200c/DOC to inhibit cancer stem cells and cancer cells by the gelatinases-stimuli nanoparticles. Biomaterials. 2013 Sep;34(29):7191-203.

36 Liu Q, Li RT, Qian HQ, Yang M, Zhu ZS, Wu $\mathrm{W}$, et al. Gelatinase-stimuli strategy enhances the tumor delivery and therapeutic efficacy of docetaxel-loaded poly(ethylene glycol)poly( $\varepsilon$-caprolactone) nanoparticles. Int $\mathrm{J}$ Nanomed. 2012;7:281-95.

37 Kim KW, Hwang M, Moretti L, Jaboin JJ, Cha YI, Lu B. Autophagy upregulation by inhibi- tors of caspase- 3 and mTOR enhances radiotherapy in a mouse model of lung cancer. Autophagy. 2008 Jul;4(5):659-68.

38 Cui F-B, Li R-T, Liu Q, Wu P-Y, Hu W-J, Yue G-F, et al. Enhancement of radiotherapy efficacy by docetaxel-loaded gelatinase-stimuli PEG-Pep-PCL nanoparticles in gastric cancer. Cancer Lett. 2014 Apr;346(1):53-62.

39 Wu F, Li R-T, Yang M, Yue G-F, Wang H, Liu $\mathrm{Q}$, et al. Gelatinases-stimuli nanoparticles encapsulating 5-fluorouridine and 5-aza-2'-deoxycytidine enhance the sensitivity of gastric cancer cells to chemical therapeutics. Cancer Lett. 2015 Jul;363(1):7-16.

40 Li X, Li R, Qian X, Ding Y, Tu Y, Guo R, et al. Superior antitumor efficiency of cisplatinloaded nanoparticles by intratumoral delivery with decreased tumor metabolism rate. Eur J Pharm Biopharm. 2008 Nov;70(3):726-34.

41 Li X, Lu X, Xu H, Zhu Z, Yin H, Qian X, et al. Paclitaxel/tetrandrine coloaded nanoparticles effectively promote the apoptosis of gastric cancer cells based on "oxidation therapy." Mol Pharm. 2012 Feb;9(2):222-9.

42 Cui FB, Liu Q, Li RT, Shen J, Wu PY, Yu LX, et al. Enhancement of radiotherapy efficacy by miR-200c-loaded gelatinase-stimuli PEGPep-PCL nanoparticles in gastric cancer cells. Int J Nanomedicine. 2014 May;9(1):2345-58.

43 Parvanian S, Mostafavi SM, Aghashiri M. Multifunctional nanoparticle developments in cancer diagnosis and treatment. Sens Biosensing Res. 2017 Apr;13:81-7.

44 Seymour LW, Ulbrich K, Wedge SR, Hume IC, Strohalm J, Duncan R. N-(2-hydroxypropyl)methacrylamide copolymers targeted to the hepatocyte galactose-receptor: pharmacokinetics in DBA2 mice. Br J Cancer. 1991 Jun; 63(6):859-66.

45 Witzigmann D, Quagliata L, Schenk SH, Quintavalle C, Terracciano LM, Huwyler J. Variable asialoglycoprotein receptor 1 expression in liver disease: implications for therapeutic intervention. Hepatol Res. 2016 Jun;46(7):686-96.

46 Julyan PJ, Seymour LW, Ferry DR, Daryani S, Boivin CM, Doran J, et al. Preliminary clinical study of the distribution of HPMA copolymers bearing doxorubicin and galactosamine. J Control Release. 1999 Feb;57(3):281-90.

47 Xu L, Xu S, Wang H, Zhang J, Chen Z, Pan L, et al. Enhancing the efficacy and safety of doxorubicin against hepatocellular carcinoma through a modular assembly approach: the combination of polymeric prodrug design, nanoparticle encapsulation, and cancer cell-specific drug targeting. ACS Appl Mater Interfaces. 2018 Jan;10(4):3229-40.

48 Devulapally R, Foygel K, Sekar T V, Willmann JK, Paulmurugan R. Gemcitabine and antisense-microRNA co-encapsulated PLGA-PEG polymer nanoparticles for hepatocellular carcinoma therapy. ACS Appl Mater Interfaces. 2016 Dec;8(49):33412-22.

49 Wang K, Kievit FM, Sham JG, Jeon M, Stephen ZR, Bakthavatsalam A, et al. Iron-oxidebased nanovector for tumor targeted siRNA delivery in an orthotopic hepatocellular carcinoma xenograft mouse model. Small. 2016 Jan;12(4):477-87.

50 Sun W, Wang Y, Cai M, Lin L, Chen X, Cao $\mathrm{Z}$, et al. Codelivery of sorafenib and GPC3
siRNA with PEI-modified liposomes for hepatoma therapy. Biomater Sci. 2017 Nov;5(12): 2468-79.

51 Mohamed NK, Hamad MA, Hafez MZ, Wooley KL, Elsabahy M. Nanomedicine in management of hepatocellular carcinoma: challenges and opportunities. Int J Cancer. 2017 Apr;140(7):1475-84.

52 Reebye V, Huang KW, Lin V, Jarvis S, Cutilas $\mathrm{P}$, Dorman S, et al. Gene activation of CEBPA using saRNA: preclinical studies of the first in human saRNA drug candidate for liver cancer. Oncogene. 2018 Jun;37(24):3216-28.

53 Setten RL, Lightfoot HL, Habib NA, Rossi JJ. Development of MTL-CEBPA: Small Activating RNA Drug for Hepatocellular Carcinoma. Curr Pharm Biotechnol. 2018;19(8): 611-21.

54 El Dika I, Lim HY, Yong WP, Lin CC, Yoon JH, Modiano M, et al. An Open-Label, Multicenter, Phase I, Dose Escalation Study with Phase II Expansion Cohort to Determine the Safety, Pharmacokinetics, and Preliminary Antitumor Activity of Intravenous TKM080301 in Subjects with Advanced Hepatocellular Carcinoma. Oncologist. 2019 Jun;24(6): 747-e218.

55 Ducreux M, Cuhna AS, Caramella C, Hollebecque A, Burtin P, Goéré D, et al. Cancer of the pancreas: ESMO Clinical Practice Guidelines for diagnosis, treatment and follow-up. Ann Oncol. 2015 Sep;26 Suppl 5:v56-68.

56 Von Hoff DD, Ervin T, Arena FP, Chiorean EG, Infante J, Moore M, et al. Increased survival in pancreatic cancer with nab-paclitaxel plus gemcitabine. N Engl J Med. 2013 Oct; 369(18):1691-703.

57 Patra CR, Bhattacharya R, Wang E, Katarya A, Lau JS, Dutta S, et al. Targeted delivery of gemcitabine to pancreatic adenocarcinoma using cetuximab as a targeting agent. Cancer Res. 2008 Mar;68(6):1970-8.

58 Trabulo S, Aires A, Aicher A, Heeschen C, Cortajarena AL. Multifunctionalized iron oxide nanoparticles for selective targeting of pancreatic cancer cells. Biochim Biophys Acta, Gen Subj. 2017 Jun;1861(6):1597-605.

59 Hoskins C, Ouaissi M, Lima SC, Cheng WP, Loureirio I, Mas E, et al. In vitro and in vivo anticancer activity of a novel nano-sized formulation based on self-assembling polymers against pancreatic cancer. Pharm Res. 2010 Dec;27(12):2694-703.

60 Hu C-MJ, Kaushal S, Tran Cao HS, Aryal S, Sartor M, Esener S, et al. Half-antibody functionalized lipid-polymer hybrid nanoparticles for targeted drug delivery to carcinoembryonic antigen presenting pancreatic cancer cells. Mol Pharm. 2010 Jun;7(3):914-20.

61 Pal K, Al-Suraih F, Gonzalez-Rodriguez R, Dutta SK, Wang E, Kwak HS, et al. Multifaceted peptide assisted one-pot synthesis of gold nanoparticles for plectin-1 targeted gemcitabine delivery in pancreatic cancer. Nanoscale. 2017 Oct;9(40):15622-34.

62 Valle JW, Armstrong A, Newman C, Alakhov V, Pietrzynski G, Brewer J, et al. A phase 2 study of SP1049C, doxorubicin in P-glycoprotein-targeting pluronics, in patients with advanced adenocarcinoma of the esophagus and gastroesophageal junction. Invest New Drugs. 2011 Oct;29(5):1029-37. 